\title{
Transcatheter Repair of Anterior Mitral Leaflet Perforation in a Patient with Mechanical Aortic Valve Using Antegrade and Retrograde Approaches: Case Report
}

Reda Abuelatta, MD1, Hesham Naeim, MD 1,2, Ahmad AlAhmadi, MD1, Saleh Al Ghamdi, MD', Osama Amoudi, MD' ${ }^{1}$, Ibraheem AlHarbi, MD ${ }^{1}$, Abdelfatah Elasfar, MD ${ }^{1,3^{*}}$

${ }^{1}$ Adult Cardiology Department, Madinah Cardiac Center, Madinah, Saudi Arabia

${ }^{2}$ Cardiology Department, Alazhar University, Cairo, Egypt

${ }^{3}$ Cardiology Department, Tanta University, Egypt

\begin{abstract}
Mitral leaflet perforations after surgical aortic valve replacement may be iatrogenic or due to endocarditis. We present a 20-year-old female who underwent surgical mechanical aortic valve replacement 8 months prior to this presentation for bicuspid severe aortic valve stenosis. She presented with acute decompensated heart failure with dyspnea and New York Heart Association (NYHA) functional class of III-IV. Transthoracic (TTE) and transesophageal echocardiography (TEE) demonstrated severe mitral regurgitation (MR) through an anterior mitral leaflet perforation. The patient refused surgical repair and percutaneous closure of the perforation was decided and performed using both antegrade and retrograde approaches. In this report, we emphasize the details and challenges of the procedure.
\end{abstract}

Copyright @ 2018 Science International Corp.

\section{Key Words}

Mitral leaflet perforation, Mechanical aortic valve, Catheter-based Mitral valve interventions

\section{Introduction}

Anterior mitral leaflet perforation complicating bicuspid aortic valve has been reported and are mostly iatrogenic or related to infective endocarditia [1]. For patients with clinical symptoms, surgical re-intervention is generally the accepted approach [2]; however, reoperation after aortic valve replacement may be associated with an increased risk of mortality and morbidity. Very few sporadic cases of percutaneous closure of perforated anterior mitral leaflet have been reported [3-5]. We describe a case of successful percutaneous closure of an anterior mitral leaflet perforation in a patient who previously had a mechanical aortic valve replacement. We present this case to emphasize the role of double antegrade and retrograde approaches through both femoral arterial and venous accesses and the challenges of the procedure.

\section{Case Report}

\section{History}

A 20-year-old female was diagnosed with bicuspid aortic valve (AV) and severe aortic stenosis (AS) complicated by infective endocarditis and mechanical AV

* Corresponding Author:

Abdelfatah Elasfar, MD

Adult Cardiology Department

Madinah Cardiac Center

Khalid Ibn Al Waleed Rd, Medina, 42351, Saudi Arabia

Tel.: +966 55691 3210; Fax: +966 14855 0481; E-Mail: elasfar_egy@hotmail.com \\ (c) 2018 Journal of Structural Heart Disease \\ ISSN 2326-4004 \\ tp://structuralheartdisease.org/
}




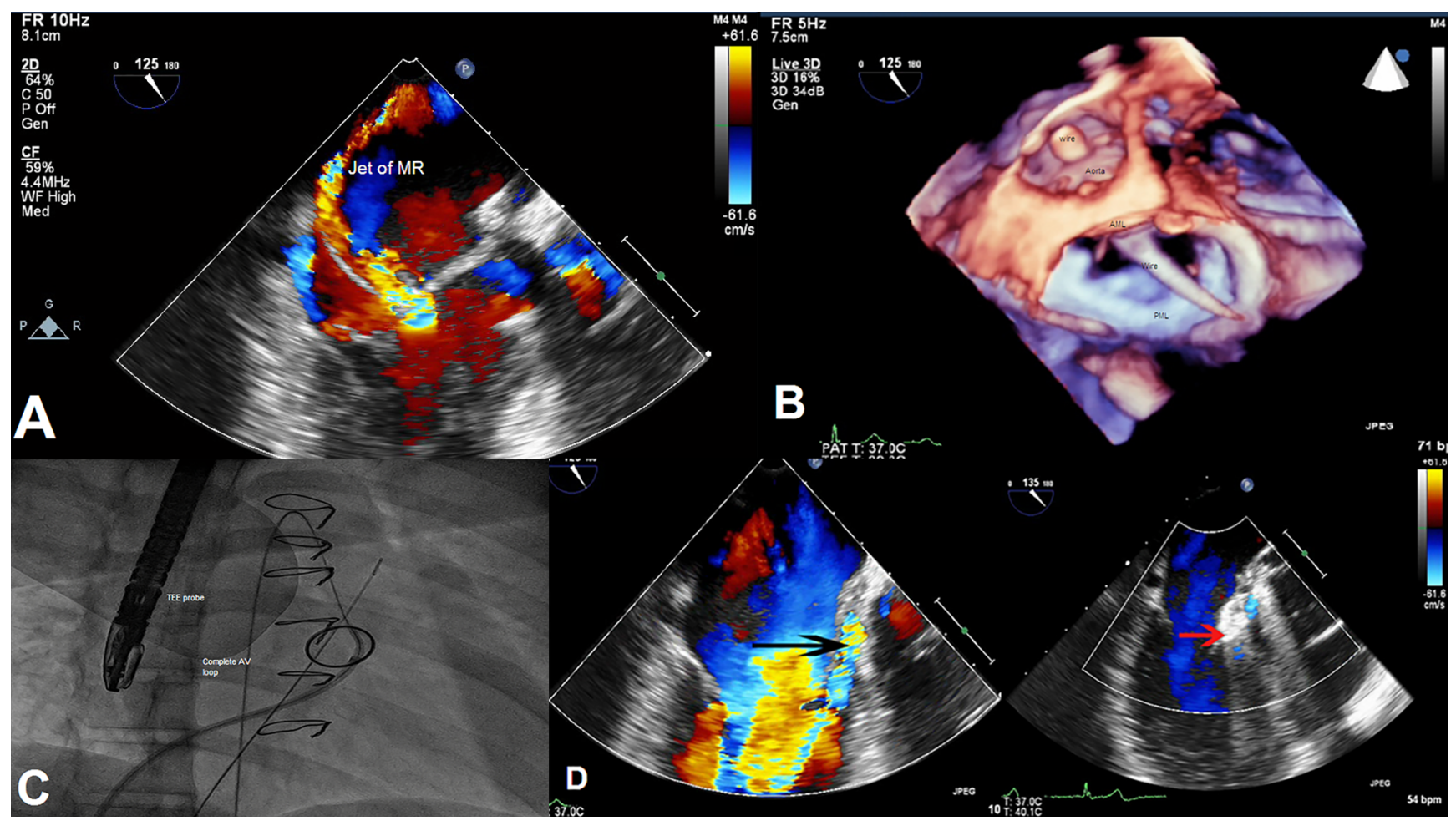

Figure 1. Panel A. TEE showing severe mitral regurgitation through the AML perforation. Panel B. 3D-TEE showing the wire passing from the AV to AML perforation. Panel C. Fluoroscopic view showing the complete arteriovenous loop and delivery sheath introduction. Panel D. 2D-TEE view during passing the mechanical aortic valve with the wire, showing moderate aorticregurgitation(left); after device implantation and removal of the wires, there is no aortic regurgitation (right).

replacement was done. Eight months later, she presented to our center with progressive SOB with NYHA class III-IV. Cardiovascular examination revealed 4/6 holosystolic murmur at the apex. Transthoracic echocardiography revealed severe mitral incompetence (Figure 1A). Transesophageal echocardiography (TEE) showed $5 \times 5 \mathrm{~mm}$ anterior mitral leaflet (AML) perforation through the $A 2$ segment with moderate pulmonary hypertension (estimated systolic pulmonary artery pressure of $50 \mathrm{mmHg}$ ). The aortic valve showed a mean gradient of $18 \mathrm{~mm} / \mathrm{Hg}$ across the AV with no valvular or paravalvular leaks. The left ventricular ejection fraction (LVEF) was $55 \%$ and the left ventricular end systolic diameter was $46 \mathrm{~mm}$. Several blood cultures were taken and they showed no bacterial growth. As the patient refused redo surgery, she was referred for a possible percutaneous closure of $A M L$ perforation.

\section{Procedure}

The procedure was performed under general anesthesia with three-dimensional TEE guidance (PHILLIPS iE33 Cardiovascular Ultrasound, USA) and periprocedural prophylactic antibiotics were given. The challenges were crossing the defect in the A2 segment, selecting the appropriate device and the AML behavior after device deployment. Very low transseptal puncture was intended to create a straight tract without tension on the AML during closure. We anticipated that crossing the defect from the LA side will be extremely difficult due to leaflet's movement away from and parallel to the crossing wire with each heartbeat. In addition, crossing through the mechanical aortic valve may carry the challenge of hemodynamic instability or mechanical disruption of the valve. Arterial and venous femoral accesses were secured and heparin was given. Transseptal access was done; tip deflectable catheter (Agilis St Jude) $8.5 \mathrm{~F}$ was introduced 


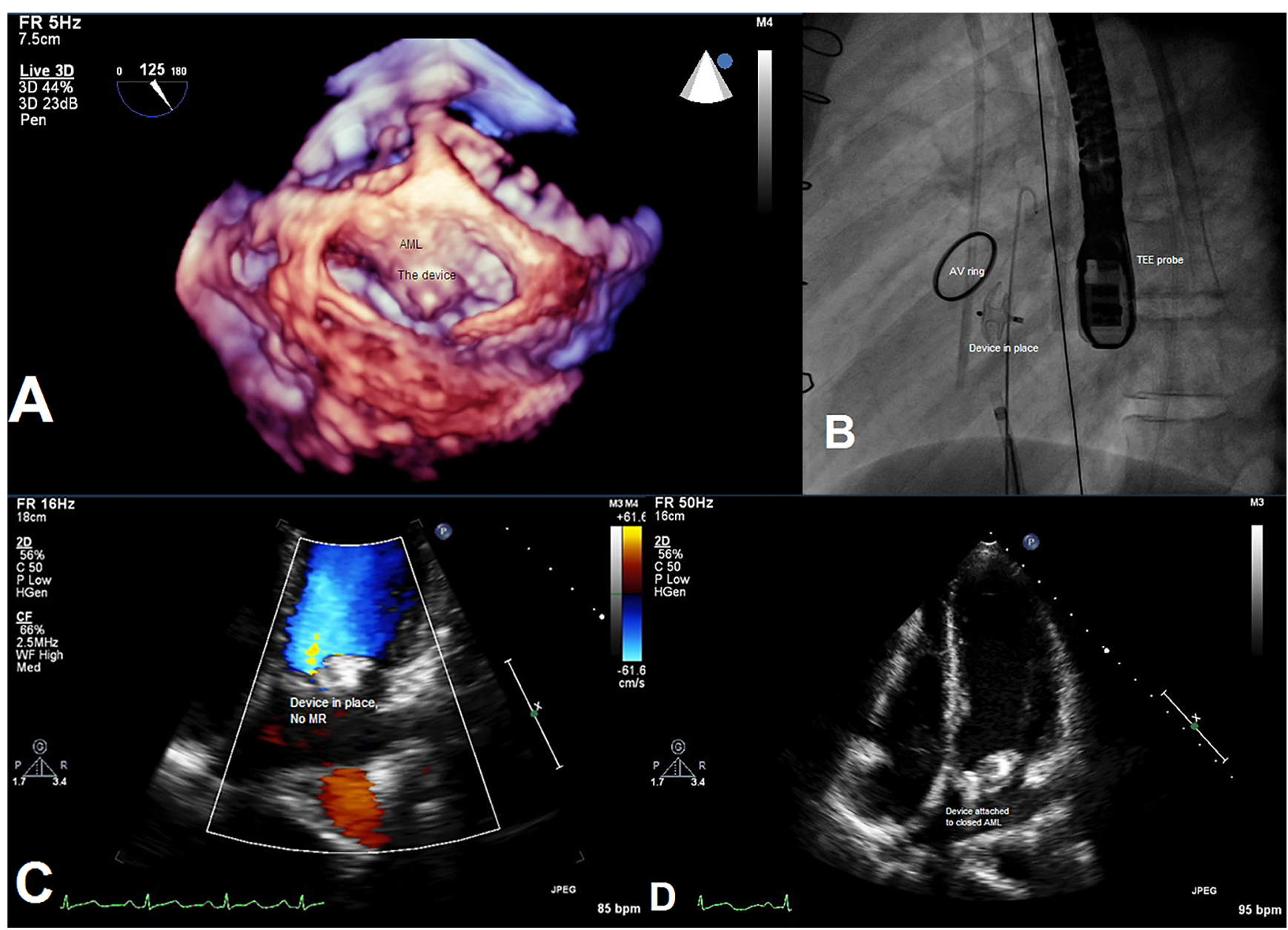

Figure 2. Panel A. 3D-TEE showing the device located within the AML sealing the perforation. Panel B. Fluoroscopic view showing the device after its release in the AML. Panel C. Colour-TTE apical view after 6 months following the procedure with no residual MR. Panel D. 2D-TTE apical view showing the device fixed in the AML 6 months after the procedure.

for effective negotiation in the LA cavity and through the anterior mitral leaflet perforation (Figure 1B, 1C).

With the help of 2 dimensional (2D) TEE at a 120-degree angle with slight clockwise rotation, the mechanical AV and the AML perforation were visualized at the same view helping to cross the defect. Real-time 3D imaging was used to monitor device implantation. Retrograde crossing using cut pigtail catheter and 0.035 "Terumo glide wire across one orifice of the aortic valve was successful, avoiding the central slit orifice (Figure 1D). The cut pigtail, with a suitable curve, successfully passed to the LV cavity then was carefully pulled back to the level of the AML, and the wire was easily oriented through the hole of the AML. This step ended by snaring the wire in the LA forming the com- plete arteriovenous (AV) loop (Figure 1C). A Tourque Vue 6F sheath (St Jude Amplatzer) crossed the atrial septum to the AML perforation and was forwarded to the ascending aorta, crossing the mechanical aortic valve with extreme caution as harm may affect the $A M L$, creating more injury or disrupting the mechanical aortic valve. The device chosen for closure needs to be light enough not to affect the AML mobility and needs to be fixed away enough from the closure line to avoid creating new MR through the normal MV orifice. We selected an atrial septal defect closure device (Amplatzer septal occluder, St Jude) size $4 \mathrm{~mm}$ with the large disc $(16 \mathrm{~mm})$ designed to be in the LV side for better stability. During and after crossing the defect, monitoring with real-time 3D-TEE imaging was 


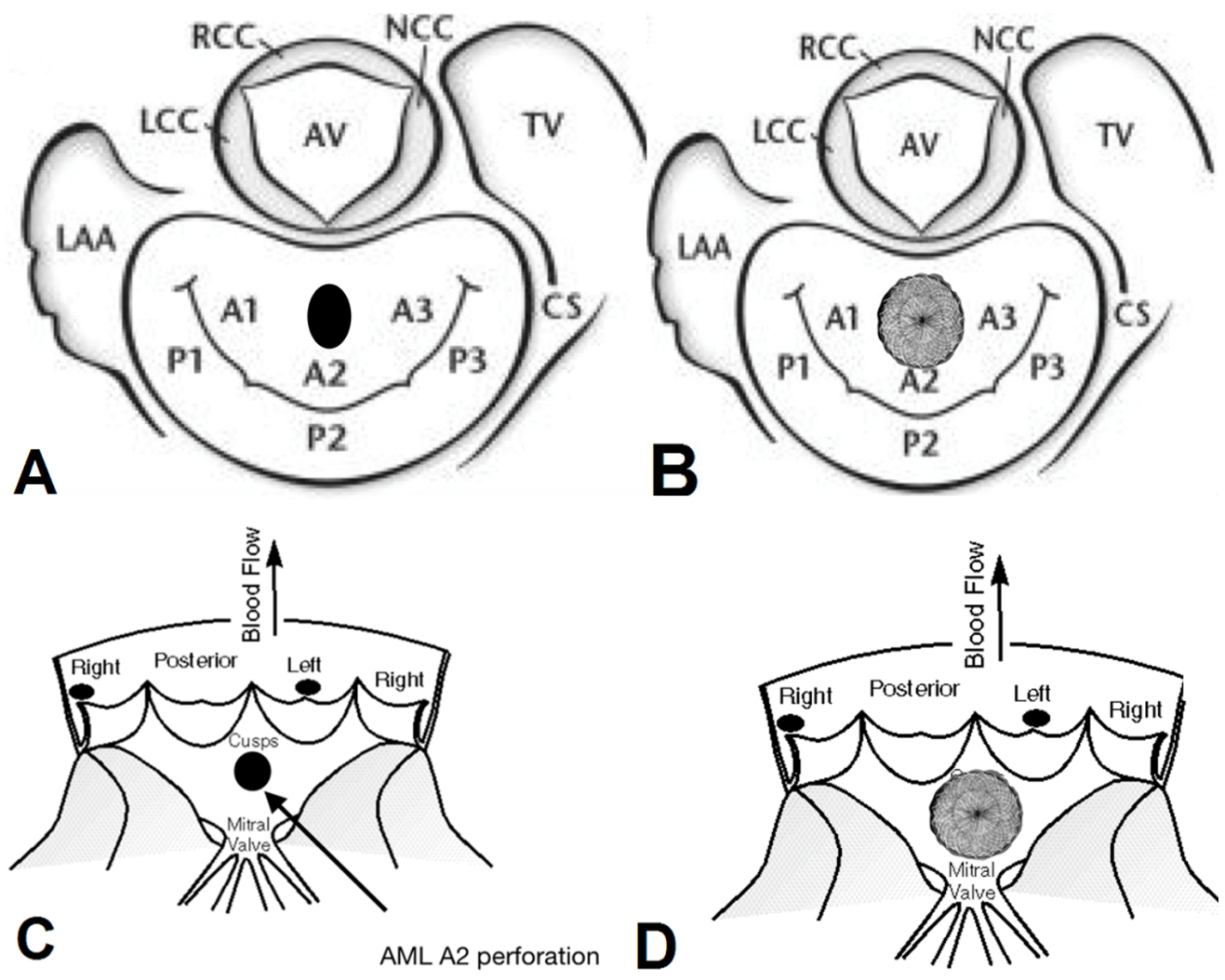

Figure 3. Panels $A$ and $C$. A cartoon showing the location of the anterior mitral leaflet perforation and its relation to the aortic valve, it was $5 \times 5 \mathrm{~mm}$ in diameter and $8 \mathrm{~mm}$ away from the mitral valve closure line. Panels $B$ and $D$. Same cartoon showing the ASD closure device in place and its relation to the MV closure line and also relation to aortic valve.

helpful through device deployment. The device was partially opened through the aortic valve then fully opened in the LVOT with good secured traction of the delivery system to close the second disc sandwiching the AML. The device showed no interference with the mitral valve closure mechanism and the anterior mitral leaflet moved freely. TEE showed no residual mitral incompetence; no diastolic gradient across the mitral valve and no LVOT systolic gradient (Figure 2A and 2B).

\section{Follow up}

The patient's clinical course was excellent as she had significant symptomatic improvement with NYHA class I and her follow-up echocardiography showed no residual MR, no diastolic mitral valve gradient and estimated systolic pulmonary artery pressure of $35 \mathrm{mmHg}$ after 6 months following the procedure (Figure 2C and 2D).

\section{Discussion}

Mitral leaflet perforations are generally rare and mostly due to infective endocarditis $[1,6]$. Other causes can be iatrogenic and would have occurred during surgery for the aortic valve, or due to autoimmune diseases like systemic lupus, erythematosus, or antiphospholipid syndrome [7]. During aortic valve surgery, anterior mitral leaflet perforation can happen due to the fibrous continuity between the anterior mitral leaflet and the aortic valve [8]. Furthermore, the middle of the anterior mitral leaflet corresponds to the anatomical location of the commissure between the left and non-coronary sinuses of the aortic valve [8]. Because of this close anatomical proximity, either of the two valves may be injured during intervention for the other [8]. In a review of the complications in 475 cases after repair of aortic valve insufficiency done by Dyck et al. [9]; they reported two cases of perforation of the base of the anterior mitral leaflet. In 
some patients, the mechanism of injury to the mitral valve anterior leaflet is aortic valve regurgitation, with the regurgitant jet being directed towards the mitral valve anterior leaflet, eroding the tissue and leaving the surface more prone to infection [8].

As endocarditis is sometimes associated, infection must be excluded in all patients with leaflet perforation. Perforations in the anterior leaflet may be the only mechanism of mitral regurgitation and if it is large, it may cause severe heart failure and warrant intervention whenever they are diagnosed [1,2].

In this reported case, multiple blood cultures drawn over two weeks were negative, and no vegetations were seen on TEE. In our case, the perforation may have been either iatrogenic, possibly because of surgical aortic valve replacement, or as a complication of the endocarditis that was diagnosed preoperatively. Surgery is the standard treatment for patients with mitral leaflet perforations [8]; but because of the higher risk related to the redo surgery and the patient's preference, percutaneous procedure was adopted.

Percutaneous closure carries multiple challenges which include crossing the leaflet perforation, which can be done from either the LA side or the ventricular side, the site of transseptal access, feasibility of crossing and negotiating the mechanical aortic valve, and how much the device can affect the closure mechanism of the mitral valve. We chose a very low septal puncture to avoid stretching the leaflet during manipulation. Then we decided to use either IM catheter or cut pigtail for negotiating the perforation from the LVOT as it was faster and easier. For the mechanical aortic valve, we avoided any excessive tension on the valve and made sure to stay away from the central slit to avoid impairment of both discs simultaneously. The best selection of the closure device was a double disc device with a distance no more than $4 \mathrm{~mm}$ between the discs, and it is best to have a larger disc towards the high-pressure chamber (LV). Also, there must be enough distance between the edge of the device and the closure line of the mitral valve. We used an atrial septal occluder device size $4 \mathrm{~mm}$ with an LV disc of $12 \mathrm{~mm}$ and waist thickness of $3 \mathrm{~mm}$. Because of the extreme difficulty of crossing, we preferred keeping a safety wire during device deployment to maintain access in case of accidental loss of the access (Figure $3 A-D)$.

In the study of Velasco S., et al. [3], they used an 8X4-mm Amplatzer Vascular Plug III with no follow up reported. In the study of Raczkiewicz S., et al. [4], they reported using a $6 \mathrm{~mm} \times 3 \mathrm{~mm}$ PLD rectangular (Paravalvular Leak Device, Occlutech). They reported five months follow up by transthoracic echocardiography with no residual regurgitation. In the study of Javed U., et al. [5], they used $5 \mathrm{~mm}$ Amplatzer atrial septal occluder.

In our case, we used an Amplatzer ASD device, however, a small Amplatzer duct occluder II, (5 to $6 \mathrm{~mm}$ with a short waist), could be another option since it's made of micronitinol with a low chance of hemolysis. It can be delivered through a much smaller delivery sheath which could minimize trauma to the mitral valve as well.

\section{Summary}

Percutaneous repair of mitral leaflet perforation caries many challenges and is only reserved for appropriately selected patients who have a high risk for surgery or in patients who refuse it. The main challenge during the procedure is the safe crossing through the defect using both the antegrade and retrograde approach. TEE guidance of the procedure is mandatory and real-time 3D is very helpful. Further research is needed to establish mid- and long-term follow up of this approach.

\section{Acknowledgment}

We acknowledge Ms. Salma Elasfar from Chatham-kent, ON, Canada, for English language revision.

\section{Conflict of Interest}

The authors have no conflict of interest relevant to this publication.

Comment on this Article or Ask a Question 


\section{References}

1. Nomeir AM, Downes TR, Cordell AR. Perforation of the Anterior Mitral Leaflet Caused by Aortic Valve Endocarditis: Diagnosis by Two-Dimensional, Transesophageal Echocardiography and Color Flow Doppler. J Am Soc Echocardiogr. 1992;5(2):195-8. DOI: 10.1016/S0894-7317(14)80553-1

2. Sareyyupoglu B, Schaff HV, Suri RM, Connolly HM, Daly RC, Orszulak TA. Safety and Durability of Mitral Valve Repair for Anterior Leaflet Perforation. J Thorac Cardiovasc Surg. 2010;139(6):1488-1493. DOI: 10.1016/j.jtcvs.2009.07.040

3. Velasco S, Larman M, Eneriz M. Percutaneous Closure of a Native Mitral Valve Perforation. Rev Esp Cardiol. 2010;63:597. DOI: 10.1016/S0300-8932(10)70121-4

4. Raczkiewicz $S$, Matejszczak-Woś $M$, Pysz $P$, Zaremba-Flis E, Smolka G, Kleinrok A. First Report of Percutaneous Closure of Anterior Mitral Leaflet Perforation Using a Paravalvular Leak Device (PLD). Postepy Kardiol Interwencyjnej. 2016;12(3):274-275. DOI: 10.5114/aic.2016.61653
5. Javed U, MD, Smith TW, Rogers JH. Percutaneous Repair of Anterior Mitral Leaflet Perforation. J Invasive Cardiol. 2012;24:134137. PMID: 22388309

6. Arat-Ozkan A, Okcun B, Mert M, Baran T, Kucukoglu S. Tear in Mitral Anterior Leaflet as a Complication of Manouguian's Procedure in a Woman With Aortic Valve Prosthesis. J Heart Valve Dis. 2004;13:630-1. PMID: 15311870

7. Bouma W, Klinkenberg T, Horst I, Hamer IJW, Erasmus ME, Bijl M, et al. Mitral Valve Surgery for Mitral Regurgitation Caused by Libman Sacks Endocarditis: A Report of Four Cases and a Systematic Review of the Literature. J Cardiothoracic Surg. 2010;5(13):1-13. DOI: 10.1186/1749-80905-13

8. Veronesi F, Corsi C, Sugeng L, Mor-Avi V, Caiani EG, Weinert L, Lamberti C, Lang RM. A Study of Functional Anatomy of Aortic-Mitral Valve Coupling Using 3D Matrix Transesophageal Echocardiography. Circ Cardiovasc Imaging. 2009;2:24-31. DOI:
10.1161/CIRCIMAGING.108.785907

9. Dyck MV, Glineur D, Kerchove LD, El Khoury G. Complications After Aortic Valve Repair and Valve Sparing Procedures. Annals of Cardiothoracic Surg. 2013;2(1):130-139. PMID: 23977571

Cite this article as: Abuelatta $R$, Naeim $\mathrm{H}$, AlAhmadi A, Al Ghamdi S, Amoudi O, AlHarbi I, Elasfar A. Transcatheter Repair of Anterior Mitral Leaflet Perforation in a Patient with Mechanical Aortic Valve Using Antegrade and Retrograde Approaches: Case Report. Structural Heart Disease. 2018;4(5):234-239. DOI: https:// doi.org/10.12945/j.jshd.2018.002.18 